\title{
Trajectories of change after a health-education program in Japan: decay of impact in anxiety, depression, and patient- physician communication
}

\author{
Min Jeong Park ${ }^{1,2}$, Joseph Green ${ }^{\text {Corresp., }}{ }^{3}$, Hun Sik Jung ${ }^{4}$, Yoon Soo Park ${ }^{5}$ \\ ${ }^{1}$ Department of Nursing, College of Nursing, Konyang University, Daejeon, South Korea \\ 2 Department of Health Communication, University of Tokyo, Tokyo, Japan \\ 3 Graduate School of Medicine, University of Tokyo, Tokyo, Japan \\ 4 College of Global Business, Konyang University, Nonsan, South Korea \\ 5 College of Medicine, University of Illinois at Chicago, Chicago, United States \\ Corresponding Author: Joseph Green \\ Email address: jgreen@gol.com
}

Background. Health education can benefit people with chronic diseases. However, in previous research those benefits were small, and reinforcement to maintain them was not effective. A possible explanation is that the benefits appeared to be small and reinforcement appeared to be ineffective because those analyses mixed data from two latent groups: one group of people who needed reinforcement and one group of people who did not. The hypothesis is that mixing the data from those two different groups caused the true effects to be "diluted."

Methods. To test that hypothesis we used data from the Chronic Disease Self-Management Program in Japan, focusing on anxiety, depression, and patient-physician communication. To identify latent trajectories of change after the program, we used growth-mixture modeling. Then, to find out which baseline factors were associated with trajectory-group membership, we used logistic regression.

Results. Growth-mixture modeling revealed two trajectories - two groups that were defined by distinct patterns of change after the program. One of those patterns was improvement followed by backsliding: decay of impact. On anxiety and depression the decay of impact was large enough to be clinically important, and its prevalence was as high as 50\%. Next, logistic regression analysis revealed that being in the decay-of-impact group could be predicted from multimorbidity, low self-efficacy, and high scores on anxiety or depression at baseline. In addition, one unexpected finding was an association between multimorbidity and better patient-physician communication.

Conclusions. These results support the hypothesis that previous findings (i.e. apparently small effect sizes and apparently ineffective reinforcement) actually reflect "dilution" of large effects, which was caused by mixing of data from distinct groups. Specifically, there was one group with decay of impact and one without. Thus, evaluations of health education should include analyses of trajectory-defined groups. These results show how the group of people who are most likely to need reinforcement can be identified even before the educational program begins. Extra attention and reinforcement can then be tailored. They can be focused specifically to benefit the people with the greatest need. 
2 Trajectories of change after a health-education program in Japan: decay of impact in

3 anxiety, depression, and patient-physician communication

4

5 Min Jeong Park ${ }^{1,2}$, Joseph Green ${ }^{3}$, Hun Sik Jung ${ }^{4}$, Yoon Soo Park ${ }^{5}$

6

$7 \quad{ }^{1}$ Department of Nursing, College of Nursing, Konyang University, Daejeon, Republic of Korea

8 2Department of Health Communication, The University of Tokyo, Tokyo, Japan

$9{ }^{3}$ Graduate School of Medicine, The University of Tokyo, Tokyo, Japan

$10{ }^{4}$ College of Global Business, Konyang University, Nonsan, Republic of Korea

$11{ }^{5}$ College of Medicine, University of Illinois at Chicago, Chicago, IL, USA

12

13

14

15 Corresponding author:

16 Joseph Green ${ }^{3},<$ jgreen@m.u-tokyo.ac.jp>

17

18 


\section{ABSTRACT}

21 Background. Health education can benefit people with chronic diseases. However, in previous

22 research those benefits were small, and reinforcement to maintain them was not effective. A

23 possible explanation is that the benefits appeared to be small and reinforcement appeared to be

24 ineffective because those analyses mixed data from two latent groups: one group of people who

25 needed reinforcement and one group of people who did not. The hypothesis is that mixing the

26 data from those two different groups caused the true effects to be "diluted."

27 Methods. To test that hypothesis we used data from the Chronic Disease Self-Management

28 Program in Japan, focusing on anxiety, depression, and patient-physician communication. To

29 identify latent trajectories of change after the program, we used growth-mixture modeling. Then,

30 to find out which baseline factors were associated with trajectory-group membership, we used

31 logistic regression.

32 Results. Growth-mixture modeling revealed two trajectories - two groups that were defined by 33 distinct patterns of change after the program. One of those patterns was improvement followed

34 by backsliding: decay of impact. On anxiety and depression the decay of impact was large

35 enough to be clinically important, and its prevalence was as high as 50\%. Next, logistic

36 regression analysis revealed that being in the decay-of-impact group could be predicted from

37 multimorbidity, low self-efficacy, and high scores on anxiety or depression at baseline. In

38 addition, one unexpected finding was an association between multimorbidity and better patient-

39 physician communication.

40 Conclusions. These results support the hypothesis that previous findings (i.e. apparently small

41 effect sizes and apparently ineffective reinforcement) actually reflect "dilution" of large effects,

42 which was caused by mixing of data from distinct groups. Specifically, there was one group with 
43 decay of impact and one without. Thus, evaluations of health education should include analyses

44 of trajectory-defined groups. These results show how the group of people who are most likely to 45 need reinforcement can be identified even before the educational program begins. Extra attention 46 and reinforcement can then be tailored. They can be focused specifically to benefit the people

47 with the greatest need.

48 

sustained. In some studies, benefits of health education have been found to last longer than 6 months (Barlow, Wright, Turner, \& Bancroft, 2005; Barlow et al., 2008; Brady et al., 2013), while in others the findings are more nuanced - some improvements do not endure (Caplin \& Creer, 2001; Clark, 2003; Franks, Chapman, Duberstein, \& Jerant, 2009; Hennessy et al., 1999; Krebs, Prochaska, \& Rossi, 2010; Lorig, Ritter, Laurent, \& Fries, 2004; Norris, Lau, Smith, Schmid, \& Engelgau, 2002). Particularly with regard to the self-management educational interventions that are used to help people reduce the impact of chronic diseases, it was recently noted that those interventions might need improvements to ensure that they have longer-lasting impact (Miller, Lasiter, Ellis, \& Buelow, 2015), which at least implicitly acknowledges that their immediate benefits can decay after their (short-lived) demonstrated period of effectiveness. To sustain these programs' benefits, reinforcement has been recommended (Clark, 2003; Green, 1977; Newman, Steed, \& Mulligan, 2009), but reinforcement has generally not been found to be useful (Glasgow, Toobert, Hampson, \& Strycker, 2002; Lorig \& Holman, 1989; Lorig, Ritter, Laurent, \& Plant, 2006; Lorig, Ritter, Villa, \& Piette, 2008; Nguyen, Carrieri-Kohlman, Rankin, Slaughter, \& Stulbarg, 2005; Riemsma, Taal, \& Rasker, 2003). which can improve health status and can increase the frequency of desirable health-related behaviors (Franek, 2013; Whitelaw, Lorig, Smith, \& Ory, 2013). While the benefits of the CDSMP are "statistically significant," some of them have also been described as minimal (Franek, 2013) or moderate (Brady et al., 2013). The program's developers attributed these

71 "modest" effect sizes to heterogeneity in the clinical and demographic characteristics of the 
72 participants (Lorig, Ritter, Laurent, \& Plant, 2006), which points to a need to understand

73 differences among participants and factors that might contribute to larger and more-sustained

74 benefits.

75 In general, treating participants as homogeneous conceals true heterogeneity. For example, 76 an intervention may be very useful in some participants, but that fact will not be recognized if 77 one examines only the average for the group as a whole (Moynihan, Henry, \& Moons, 2014). In 78 addition, important heterogeneity in treatment effects can occur not only across groups but also over time. Sustained improvement in some participants could obscure relapse in others.

80

82

83

84 85

Reinforcement given to all can appear to be ineffective, even if it is quite useful to some.

In previous research, subsets of participants were defined by socio-demographic

characteristics, personality factors, etc., and not by patterns of change after the intervention

(Franks et al, 2009; Harrison et al., 2012; Jerant, Chapman, Duberstein, \& Franks, 2010; Reeves

et al., 2008; Swerissen et al., 2006; Smeulders et al., 2010). In contrast, LW Green (1977)

discussed five distinct patterns (i.e. trajectories) of change after health education. He referred to the pattern in which good outcomes do not endure as decay of impact (also sometimes called relapse or backsliding). Its essence is simple: deterioration after improvement. Outside of health education, a similar concept is used commonly in research on the effectiveness of treatments for addiction (Hendershot et al., 2011; Menon \& Kandasamy, 2018). Within the field of health education, Green (1977) may have been the first to point out that decay of impact can cause the benefits of an intervention to appear to be larger or smaller than they really are, depending on when the outcome is measured. That difference between the apparent change and the real change can be minimized only by measuring the outcome repeatedly over a relatively long period of time. Hennessy et al. (1999) measured self-efficacy for condom use 5 times during 1 year after 
95 an HIV-prevention educational program, and they found clear evidence of decay of impact 3

96 months after enrollment in the program. Caplin and Creer (2001) identified various

97 characteristics of people with decay of impact 7 years after an asthma self-management program:

98 For example, the people with decay of impact had initially been less self-motivated to participate

99 in the program. Nonetheless, with only a few exceptions, over more than 40 years since Green

100 (1977) described decay of impact in health education, that concept seems to have received little

101 attention from researchers. Stated simply, decay of impact as a person's pattern of change after

102 chronic-disease self-management education has been studied only very rarely. But knowing the

103 magnitude of decay, and knowing when and in whom it occurs would be very useful in

104 evaluating a health-education program's effectiveness, and also in targeting interventions.

105 Information about decay of impact can give planners an objective basis for deciding whether

106 reinforcement is needed, when it is needed (Hennessy et al., 1999), and who is most likely to

107 need it (Park, Green, Ishikawa, \& Kiuchi, 2012).

108 Taking seriously the possibility of decay of impact entails defining groups by their

109 patterns of change, i.e. the trajectories of their outcomes during follow-up. Using data collected

110 in two waves, Nolte, Elsworth, Sinclair, \& Osborne (2007) may have been the first to study

111 groups defined by their change after health education. An extension from patterns defined using

112 two waves of data to those defined using four was published a few years later (Park et al., 2013).

113 However, without the use of a well-established method for analyzing longitudinal data, doubts

114 remain regarding different trajectories in outcomes. In this current study, using data collected

115 before and after the CDSMP we applied Growth-Mixture Modeling (GMM) to empirically

116 identify latent groups that were characterized by their patterns of longitudinal change, and we

117 subsequently used logistic regression to identify baseline factors contributing to group 
118 membership (Cook, Karriker-Jaffe, Bond, \& Lui, 2015; Hibbard, Mahoney, Stock, \& Tusler,

119 2007; Muthén, 2004; Rabe-Hesketh, Skrondal, \& Pickles, 2004; Ram \& Grimm, 2009). This

120 approach allows more granularity in evaluating the program's effects and more accuracy in

121 assessing needs for reinforcement.

122 Many outcomes of the CDSMP have been studied (Lorig et al., 1996), but here we

123 focused only on symptoms of anxiety, symptoms of depression, and the use of proactive

124 techniques to improve patient-physician communication. Thus, the focus is not on the

125 longitudinal change in participants' chronic illnesses themselves (with the possible exception of

126 the small number of participants who had clinical depression). Trajectories of change in anxiety

127 and depression could reflect changes in the application of coping skills learned in the CDSMP,

128 while patient-physician communication is of course a health-related behavior. We chose those

129 three outcomes because all of them are relevant in many different chronic medical conditions,

130 and thus the results will be important to a large proportion of all CDSMP participants. Also, one

131 area on which the CDSMP focuses specifically is learning and practicing better patient-physician

132 communication.

133

METHODS

\section{Participants}

In this study, we focused on a particular population: adults in Japan who have at least one

136 chronic medical condition and who participated in the CDSMP. In this implementation of the

137 CDSMP in Japan, the program accommodated people who were community-dwelling, were able

138 to attend weekly group meetings, and were able to communicate easily in Japanese. As in all

139 implementations of the CDSMP, the participants had various different chronic medical

140 conditions. The population of CDSMP participants in Japan consists only of adults (defined as 
141 people who were at least 18 years old), but it is otherwise diverse with respect to age, duration of

142 chronic illness, and other socio-demographic and clinical characteristics (Appendix 1).

143 CDSMP participants were recruited through public service centers, outpatient clinics of

144 hospitals, and the Internet homepage of the Japan Chronic Disease Self-Management Association

145 (Japan Chronic Disease Self-Management Association, 2018). Some participants also found out

146 about the program through friends or acquaintances. Participation was voluntary. All people who

147 met the conditions described above comprised the population of interest. The study itself

148 involved a census (not a sample) of that population.

\section{The program}

150

Based on self-efficacy theory (Bandura, 2019), the program aims to build the

151

participants' skills in six areas: 1) handling pain, fatigue, frustration, and isolation, 2) exercising

152

to maintain and increase strength, endurance, and flexibility, 3) using medications appropriately,

153

4) improving communication with friends, family, and healthcare professionals, 5) achieving and

154

maintaining proper nutrition, and, 6) evaluating new therapies (Chronic Disease Self-

155

Management Program, 2018). Skills in those areas were taught and practiced during group-

156 discussion sessions that were held once each week for six weeks. Each group had two lay

157 facilitators who had undergone approximately 35 hours of training. A textbook was used as the

158 reference for the program's content (Lorig, Holman, Sobel, Laurent, \& González, 2001).

159 Measures

160

Demographic and clinical information were collected using self-administered

161 questionnaires. Also included in the questionnaires were scales to measure health status, health-

162 related behaviors, psychological variables, etc. Those included self-efficacy to manage chronic

163 health conditions (on a 0-to-60 scale, coefficient alpha $=0.92$ ) and the Hospital Anxiety and 
164 Depression Scale (HADS, Matsudaira et al., 2009). The HADS asks about symptoms of anxiety

165 and of depression in the past week. Possible total scores on both the anxiety scale and the

166 depression scale range from 0 to 21 , with higher scores reflecting more symptoms and more

167 frequent symptoms. Coefficient alpha for the depression scale of the HADS was 0.73 and for the

168 anxiety scale it was 0.84 . Also included was a 3-item scale to measure communication with

169 physicians, with possible total scores ranging from 0 to 15 (coefficient alpha $=0.78$ ). Higher

170 scores reflected more frequent use of proactive methods for good patient-physician

171 communication (Lorig et al., 1996).

\section{Study design and timing of measurements}

173 Data were collected four times over one year. Baseline data were collected before the

174 first group-discussion session. Follow-up questionnaires were sent by postal mail 3, 6, and 12

175 months later. A post-paid envelope addressed to the research office was included for returning

176 the questionnaire. To ensure that data collection was as complete as possible, one of the

177 researchers (MJP) was available to speak directly with participants and answer their questions

178 about the study. In addition, a reminder postcard was sent whenever a follow-up questionnaire

179 was not received by return mail within two weeks.

\section{Analyses}

181 To allow detection of decay of impact, the analyses were done using data from

182 participants who provided at least three waves of data $(456 / 643 ; 71 \%)$. Unconditional quadratic

183 growth curves (Rabe-Hesketh, Skrondal, \& Pickles, 2004) and conditional growth curves were

184 fit using the four-wave data with all participants. Time-variant (self-efficacy) and time-invariant

185 covariates (gender, educational status, partnered status, number of diagnoses, and history of

186 illness) were included in the growth-curve analysis, with anxiety, depression, and 
187 communication as outcomes, to examine baseline differences and interactions over time.

188 Quadratic terms were specified on "time" by including a squared time (i.e. time x time) term.

189 Interaction terms between time-invariant covariates and time were included to examine factors

190 contributing to longitudinal changes.

191 GMM with quadratic growth curves were fit for 2, 3, and 4 latent groups. Posterior mode

192 estimation, a partly Bayesian approach, was used to obtain consistent parameter estimates and to

193 avoid boundary estimation issues (Park, Xing, \&, Lee, 2018; Huang \& Bandeen-Roche, 2004).

194 Moreover, to obtain optimal estimation, we began with 100 sets of starting values and used a

195 combination of expectation-maximization algorithm followed by Newton-Raphson to obtain

196 parameter estimates and model fit indices. Finally, we examined the Jacobian matrix to be of full

197 rank to ensure local identification of results. This process was repeated for each growth-mixture

198 model allowing for the different latent groups. We decided on the final number of groups after

199 examining the relative fit (Bayesian Information Criterion [BIC]) and absolute fit (Proportion

200 Correctly Classified $\left[P_{c}\right]$ based on posterior probability). Multiple logistic regression was used to

201 identify factors contributing to trajectory-group membership. Of the 456 participants whose data

202 were analyzed by GMM here, 369 were included in a previous non-GMM analysis (Park et al.,

203 2013). Data were analyzed with Latent Gold 5.1 (Belmont, MA), Stata 14 (College Station, TX),

204 and JASP (https://jasp-stats.org/).

205 Ethics

206 This study was approved by the University of Tokyo (number 1472-(2), Research Ethics

207 Committee, Graduate School of Medicine). Participation in the CDSMP and in this research

208 were voluntary. Informed consent was obtained in writing from all participants before the study 209 began. 


\section{RESULTS}

\section{1. The participants}

212 Data from 456 participants were analyzed. Among them, 79\% were women, $48 \%$ were

213 college educated, $52 \%$ were partnered (married or living with someone), and $47 \%$ had more than 214 one chronic condition. Details of multimorbidity are in Appendix 1.

\section{2. All participants considered together}

In the analysis with all participants considered together (Table 1), for the first six months

217 communication with physicians increased, while both anxiety and depression decreased.

218 However, by the end of the follow-up year all three outcomes had begun changing back toward

219 their baseline values. That is, the initial improvements appeared to be followed by at least some 220 backsliding.

221 Similarly, the growth-curve analysis also indicated that change over time was curvilinear

222 for all three outcomes: $p$ values for the quadratic terms for anxiety, depression, and

223 communication were $0.001,0.038$, and 0.002 , respectively (Table 2 ). In addition, higher self-

224 efficacy at baseline was associated with less anxiety, less depression, and better communication 225 with physicians.

226 Also associated with both anxiety and communication was the number of diagnoses, and 227 the regression coefficients for both were positive (Table 2). That is, the participants with more 228 comorbid conditions had greater anxiety at baseline. It is noteworthy that the participants with 229 more comorbid conditions also had better baseline scores on the scale measuring communication 230 with physicians. These associations did not change over time, as evidenced by the small

231 coefficients for the terms representing interaction with time. 


\section{3. Groups defined by their trajectories}

233 For all three outcomes, the GMM results were similar: The BIC and the $P_{c}$ both led to the

234 conclusion that the best-fitting models were those with two groups (Appendix 2).

235 For each outcome, those two groups began from substantially different baseline scores

236 (Figure 1). Also for each outcome, one group changed very little throughout the follow-up period

237 while the other changed noticeably within the first six months of follow-up, and then it reversed

238 course back toward the baseline value (Figure 1). That is, on each outcome some participants

239 were in a decay-of-impact group and the others were not. About half of the participants were in

240 the decay-of-impact group: anxiety $45.6 \%$, depression $50.7 \%$, and communication with

241 physicians $46.3 \%$.

242 Participants who had decay of impact on one of the two mental-health outcomes (anxiety

243 or depression) were also likely to be classified as having decay of impact on the other one (Phi $=$

244 0.508, Appendix 3). However, participants who had decay of impact on one of the two mental-

245 health outcomes were no more or less likely to have decay of impact on communication $(\mathrm{Phi}=$

2460.095 and 0.043, Appendix 3).

247 On the two mental-health outcomes, the decay-of-impact group was the group with worse

248 baseline status: more symptoms, and more-frequent symptoms, of anxiety and depression. In

249 contrast, on communication with physicians the decay-of-impact group was the group with better

250 baseline status: more frequent use of the three specified methods for good patient-physician

251 communication. Also, by the end of the follow-up year the anxiety and depression scores had

252 decayed back to their respective baseline levels, whereas on communication the decay trajectory

253 was clear but the scores did not return to the baseline level, in other words the decay itself was

254 smaller on communication with physicians than on the mental-health outcomes (Figure 1). 


\section{4. Contributors to group membership (Table 3)}

256 For all three outcomes, self-efficacy at baseline was associated with group membership.

257 Participants with higher self-efficacy were more likely to be in the group with lower anxiety at

258 baseline, in the group with lower depression at baseline, and in the group with better

259 communication at baseline.

260 There were no noteworthy associations between group membership and gender, civil

261 status (i.e. partnered or not), or the number of years of chronic-disease history. College education

262 was associated with group membership on only one of the three outcomes (anxiety).

263 For anxiety and for communication with physicians, the number of diagnoses was also

264 associated with group membership. Regarding anxiety, participants with more diagnoses were

265 more likely to be in the group with higher (i.e. worse) scores at baseline and subsequent decay of

266 impact. Regarding communication, participants with more diagnoses were more likely to be in

267 the group with higher (i.e. better) scores at baseline and subsequent decay of impact.

\section{DISCUSSION}

\section{All participants}

270 When all participants were considered together, all three outcomes improved over the

271 first six months. That improvement was followed by a small deterioration. Thus, even from the

272 least-detailed analyses, some decay of impact was evident (Table 1). That interpretation is

273 supported by the results of the growth-curve analyses: change over time was curvilinear

274 (quadratic) for all three outcomes (Table 2).

275 The growth-curve analyses also showed that higher self-efficacy at baseline was

276 associated with less anxiety, less depression, and better communication with physicians, which is

277 consistent with the theoretical basis of the CDSMP (Lorig \& González, 1992). 
Also evident at this level of analysis were associations with multimorbidity. Having more 279 diagnoses was associated with more anxiety, more depression, and better communication with 280 physicians. Of those three findings, the first two might be expected, but the third is particularly 281 interesting. It is also reflected in the analyses of membership in trajectory-defined groups, and so 282 we will discuss it below.

283 Findings from GMM: groups defined by their trajectories

For all three outcomes the results of GMM were consistent: Among all of the models in saying that GMM revealed two latent groups among these participants in the CDSMP. In some circumstances practical considerations could override the conclusions from those statistical criteria, as described in Appendix 2.

As noted above in the Introduction, in some previous studies, subsets of CDSMP participants were defined a priori and with reference to theory. In contrast, groups identified by

291 GMM are empirical, as is each participant's group membership. We note that the GMM 292 approach can lead to testable hypotheses (regarding multimorbidity, as described below), and it

293 can be used to answer important questions about whether similar phenomena also occur among 294 other groups and in different settings.

\section{Factors affecting self-management}

These findings are not inconsistent with the five categories of factors affecting selfmanagement that were identified by Schulman-Green et al. (2016). It is noteworthy that the CDSMP does address factors in all five of Schulman-Green's categories: self-efficacy (which is

299 in the category of "personal/lifestyle characteristics"), problem-solving to cope with symptoms 300 (in the category of "health status"), social support via the CDSMP group sessions (in the 
301 category of "resources"), action plans for ensuring proper nutrition (in the category of

302 "environmental characteristics"), and proactive patient-physician communication (in the

303 category of "health care system").

304 With regard to the decay of impact, we note that Table 3 shows associations of trajectory-

305 group membership with two factors that were identified by Schulman-Green, et al. (2016) as

306 influencers of self-management. Those two factors are self-efficacy (in their "personal/lifestyle

307 characteristics" category) and the number of diagnoses (i.e. comorbidity, in their "health status"

308 category). In addition to those two, the baseline level of patient-physician communication is in

309 Schulman-Green's category of "health care system." It is quite possible that factors in Schulman-

310 Green's two other categories ("resources" and "environmental characteristics") also affect the

311 decay of impact, and we believe this is an important topic for future research.

312 Mental health, and reinforcement

313 For both mental-health outcomes, the trajectory-defined groups differed in their baseline

314 status and in their pattern of change after the program. Regarding anxiety, approximately half of

315 the participants were in a group that began with relatively good scores, and they improved very

316 gradually over the following year. In contrast, the other half were in a group that began from a

317 high-anxiety baseline. That second group improved over the first 6 months, but by the time of the

318 12-month follow-up it had returned to its baseline level, and thus we refer to the latter group as

319 the decay-of-impact group. The same was true with regard to depression.

320 Dichotomization is undoubtedly dangerous (Harrell, 2019), and yet HADS scores are

321 used to separate people into categories of anxiety and depression severity. In Japan, the HADS

322 threshold score separating non-cases from possible and probable cases was 9 (Matsudaira et al.,

323 2009). The decay-of-impact trajectories on both anxiety and depression crossed that threshold 
324 twice - first during the improvement occurring soon after the intervention ended, and then again

325 about 8 months later during the decay back toward the baseline value (Figures $1 \mathrm{a}$ and $1 \mathrm{~b}$ ).

326 Therefore, to the extent that the threshold of 9 is useful, both the improvement measured soon

327 after the CDSMP and also the deterioration measured near the end of the follow-up year were

328 clinically important.

“Average therapeutic trial results can mislead" (Moynihan et al., 2014), but GMM

330 provides more detail than average results. Here GMM showed that only some of the participants

331 had decay of impact. At least on anxiety and depression, both the existence of a decay-of-impact

332 group and the movement of that group between clinical categories support the idea that follow-

333 up interventions - reinforcement - should be offered to some of the participants. Had

334 reinforcement been given to all, it is unlikely that those in the group without decay of impact

335 would have benefitted from it, simply because they already had almost no psychological distress

336 - almost no room to improve. Rather than being expended on all of the participants, the

337 resources used to implement reinforcement should be saved for the people who need it, to help

338 them maintain their newly-improved status or perhaps improve further.

339 The present findings show how the small effect sizes and null results in published studies

340 of reinforcement could be underestimates. Specifically, when all participants are considered

341 together (Table 1), then any benefits to a group with decay-of-impact (Figure 1) will become at

342 least more difficult to detect, and possibly even completely effaced, as they are mixed with the

343 much smaller (or even zero) benefits to the non-decay group. This dilution is clearly shown in

344 the difference between Table 1 and Figure 1. Table 1 shows only a very small decay of impact,

345 because all participants were considered together, but Figure 1 shows substantial decay of impact

346 because GMM separated the participants into two trajectory-defined groups as it "unmixed" the 
347 data. If one were to look at Table 1 alone, one could easily conclude that the benefits of the

348 program and also the decay of impact (i.e. the need for reinforcement) were both very small. But

349 that would be another example of how average results can mislead (Moynihan et al., 2014).

350 Figure 1 shows that almost half of the participants had both important benefits and important 351 decay of impact, and the difference between Table 1 (the diluted results) and Figure 1 (the post-

352 GMM results) shows how mixing the data from two different groups caused important effects to 353 be diluted.

Testing the effects of reinforcement is reasonable, but only in those whom reinforcement

355 could benefit: the decay-of-impact group. To identify that group at baseline, i.e., even before the

356 CDSMP begins, the present findings offer two potential criteria: a high baseline score (greater

357 distress) on the mental-health outcome of interest, and a low level of self-efficacy. With regard

358 to anxiety, a third criterion could be multimorbidity.

\section{Communication with physicians}

360 Similar to the results described above for mental health, regarding communication with

361 physicians GMM revealed two groups, each comprising about half of the participants, and those

362 two groups began from noticeably different baselines. One group $(n=245)$ started from a very

363 low baseline communication score (about 4 points) and it changed very little over the following

364 year (Figure 1). This could well indicate an unmet need. Specifically, by the standard implied in

365 the patient-physician communication scale, for those 245 participants substantial improvement

366 after the baseline measurement was possible, but it did not occur. This leads to at least three

367 research questions: (1) Are some participants in fact satisfied with a "low" level of

368 communication? (2) Was the program implemented as well as possible? and (3) Even if the 
369 implementation was good, would those 245 participants have benefitted from a more-intensive

370 intervention with an even-greater emphasis on practicing communication skills?

371 Also noteworthy are the three criteria that might be used to pre-emptively identify the

372 participants who are most likely to need communication-skill practice: a low communication

373 score at baseline, low self-efficacy, and unimorbidity.

374 Multimorbidity

375 The participants with more diagnoses had better communication scores in the initial

376 growth-curve analysis (Table 2), and they were more likely to be in the trajectory-defined group

377 that had better communication scores throughout the year (Table 3 ). While the CDSMP has been

378 found to be particularly useful to people with multiple diagnoses (Harrison et al., 2012), here

379 multimorbidity was associated with a desirable health-related behavior even at baseline. To

380 address the apparent connection between having multiple diagnoses and communicating well

381 with physicians, we begin by noting that the communication scores reflect how often the

382 respondents do the following three activities: making a list of questions to ask one's physician

383 during clinic visits; asking one's physician about things that one wants to know or does not

384 understand regarding one's treatment; and discussing (with one's physician) personal problems

385 related to one's medical condition. The people with multiple diagnoses probably had more

386 experience being in health-related situations that were difficult to manage. To deal with those

387 difficulties, perhaps they began writing lists of questions, asking for clarification, and discussing

388 personal issues related to their diseases, simply because their health conditions were so complex.

389 We hypothesize that at least some of the people with multimorbidity had become accustomed to

390 doing those activities, and therefore in the domain of patient-physician communication they had

391 already become "expert patients" (Reeves et al., 2008) by the time the study began. To the extent 
392 that better patient-physician communication results in better clinical care, this connection

393 between multimorbidity and good communication could account at least in part for the

394 documented association of multimorbidity with higher-quality care (Min et al., 2007).

395 Other factors could also be important. For example, self-selection might have played a

396 role. After all, participation in the program was voluntary (as it is worldwide). Personality can

397 moderate the effects of the CDSMP (Franks et al., 2009; Jerant et al., 2010), and perhaps it also

398 affects one's decision to participate. Among all of the eligible people with multimorbidity, those

399 who are less "conscientious" and less interested in self-managing their conditions would not

400 often make lists of questions, etc., and they might not have found the CDSMP to be attractive

401 and thus would not have participated. In contrast, the CDSMP might appeal to people like the

402 highly-proactive communicator with 8 chronic conditions who was described by Haslam (2015).

403 People with multiple diagnoses who take initiative in self-managing their condition(s) by writing

404 lists of questions, etc. could be over-represented among the program's participants.

405

As explanations of the association between multimorbidity and good patient-physician

406 communication, both the self-selection hypothesis and the already-an-expert-patient hypothesis

407 remain to be tested, and of course they are not mutually exclusive: both could be true.

408 Generalizability

409 Because this study involved not a sample but a census of the population of interest, the

410 common concerns about generalizability are not strictly applicable. Chronic-disease self-

411 management interventions have been implemented in many countries, but the country-specific

412 differences in those implementations are important (O'Connell, McCarthy, \& Savage, 2018), and

413 we do not claim that the results shown in Figure 1, Table 3, etc. necessarily apply to CDSMP

414 participants outside Japan. Nonetheless, we do suggest that the approach and the methods 
415 employed here could be quite useful in evaluations of the CDSMP and of other health-education

416 programs worldwide. As a minimum, evidence explaining why effect sizes appear to be small is

417 important wherever the CDSMP is used. In addition, a generalized application of GMM and

418 similar methods would ground the understanding of these programs' effects more firmly in the

419 empirical reality of trajectory-defined groups. Related analytic methods may also be useful,

420 including methods that allow analyses of individual participants' trajectories (Kozlowski, Pretz,

421 Dams-O'Connor, Kreider, \& Whiteneck, 2013). A generalized application of the approach and

422 the methods used in the present study would also help to meet the current need for research on

423 long-term maintenance of self-management skills (Miller, Lasiter, Ellis, \& Buelow, 2015).

424 Cost-effectiveness

425 Cost-effectiveness analyses of the CDSMP indicate that it results in net savings (Ahn et

426 al., 2013). Its cost-effectiveness has also been demonstrated through analysis of the incremental

427 cost-effectiveness ratio per quality-adjusted life year (Basu et al., 2015). Formal cost-

428 effectiveness analyses of the prediction of CDSMP trajectory-group membership are beyond the

429 scope of the present study. Nonetheless, it is reasonable to expect the costs to be low.

430 Specifically, for the three outcomes of interest here, the present results indicate that predicting

431 trajectory-group membership might require completion of only the 14-item HADS, the 6-item

432 self-efficacy scale, the 3-item patient-physician communication scale, and a question-item

433 regarding multimorbidity - all at baseline only. The participants already fill out registration

434 forms before the start of the first CDSMP group session. If the burden on the participants must

435 be strictly minimized, then the necessary information could be gathered only once (at baseline),

436 and it would take only a few extra minutes. 
While the costs would be expected to be low, the effectiveness (benefits) could be high,

438 because there is potential for prevention or minimization of the decay of impact. Specifically, as

439 shown in Table 3 and in Figure 1, baseline information might be used to predict who among the

440 participants will have decay of impact, and thus who is most likely to benefit from extra attention

441 during the program and from reinforcement after the program. This could strengthen the

442 empirical foundation for decisions about cost-effective allocation of those educational resources.

\section{Limitations}

444 The four waves of data collection over one year were more than enough to allow

445 detection of decay of impact, but more frequent measurement and longer follow-up would of

446 course be useful.

447 The number of diagnoses was self-reported. While we would have preferred to use

448 medical records, for many chronic conditions self-reported diagnosis is accurate enough for

449 research (Karison et al., 1999; Wada et al., 2009). The diagnosis of clinical depression was found

450 by Sanches-Villegas et al. (2008) to be over-reported, with a true-positive percentage of $74.2 \%$.

451 In that study, among 62 people who self-reported a diagnosis of depression, 46 were found to be

452 true positives according to the SCID-I $(46 / 62=0.742)$. It is possible that in the present study a

453 similar percentage of those who self-reported that they had been given a diagnosis of depression

454 would in fact have met the SCID-I criteria for depression. In that case, the number of true

455 positives would be estimated to be approximately 19 rather than the 26 who did self-report that

456 they had been given a diagnosis of depression (Appendix Table A1C): $0.742 \times 26=19.3$. This is

457 unlikely to have had a large effect on the main results or conclusions. The logistic regression

458 analysis did identify the number of diagnoses as a predictor of trajectory-group membership

459 (Table 3), but any effect of over-reporting of depression on that result was probably very small. 
460 Specifically, $26-19=7$, and $7 / 456=0.015$. Thus, self-reporting of a diagnosis of depression

461 might have caused the number of participants with depression to have been over-estimated by

462 approximately 7 , which is $1.5 \%$ of the total. In addition, it is important to remember that the

463 number of diagnoses was not included in the GMM analysis, so any inaccuracy or imprecision in

464 that number did not affect the GMM results (Figure 1 and Appendix 2). Also, the presence or

465 absence of any self-report of any specific diagnosis (depression, etc.) was not included in the

466 GMM analysis, so any inaccuracy or imprecision in those data also did not affect the GMM

467 results.

468 Previous knowledge gaps, new knowledge, and applications

469 One approach to the presentation of research is to explicate knowledge gaps, how those

470 gaps have been "filled," and the practical consequences thereof. With that in mind, we give such

471 information below.

$472 \quad$ Knowledge gap 1: The benefits of the CDSMP appear to be small (and reinforcement

473 appears to be ineffective), but the reason is not well understood. Filling knowledge gap 1, we

474 found how mixing of data from two distinct trajectory-defined groups can make the overall

475 benefits of the CDSMP appear to be small even though they are relatively large for some

476 participants. Also, one of those two trajectory-defined groups was characterized by decay of

477 impact. Given the new information obtained in filling that first gap, another knowledge gap

478 became clear.

479 Knowledge gap 2: Criteria for identifying people who are likely to have decay of impact

480 are not known. Filling knowledge gap 2, we found that the people who are most likely to have

481 decay of impact can be identified using baseline self-efficacy and the number of diagnoses 
482 (Table 3). Also, people who eventually had decay of impact were those with higher baseline 483 scores on the outcome of interest (Figure 1).

484

Applications of new knowledge: This new knowledge can be useful in at least two ways.

485 First, it can be used to tailor reinforcement to the participants who are most likely to need

486

reinforcement, which could increase cost-effectiveness. Second, it can be used to establish two

487

new goals for CDSMP implementation and two new criteria for CDSMP evaluation: a low

488

prevalence of decay of impact, and a small magnitude of decay of impact (Park et. al, 2012).

489 Considerations regarding theory

490

As noted above, the theoretical foundation of the CDSMP is centered on the concept of

491

self-efficacy (Bandura, 2019). In light of the present results, other theories and conceptual

492 frameworks may also be useful, especially those emphasizing long-term outcomes (Miller,

493 Lasiter, Ellis, \& Buelow, 2015). For example, the field of relapse prevention after treatment for

494 substance abuse has well-studied theories and practices (Hendershot, Witkiewitz, George, \&

495 Marlatt, 2011; Menon \& Kandasamy, 2018; Witkiewitz \& Marlatt, 2004), some of which might

496 be adapted to inform strategies for preventing decay of impact after chronic-disease self-

497 management education. In addition, consideration should be given to the difference between

498 changing health-related behavior and maintaining a new health-related behavior for long-term

499 benefits (Rothman, 2000; Sciamanna et al, 2011; Joseph et al., 2016; Giacobbi, 2016).

500 Conclusions

501

GMM exposed two trajectory-defined groups, and the CDSMP clearly benefitted one

502 group more than the other. However, the group that benefitted also had substantial decay of

503 impact, and thus needed reinforcement. The decay-of-impact group comprised almost half of the

504 participants. At baseline (i.e., before the program began), the participants most likely to need 
505 reinforcement were those with multimorbidity, those with low self-efficacy, and those who were 506 clinically anxious or depressed.

507 Once the participants who are likely to have decay of impact are identified, extra

508 attention and reinforcement can then be tailored. They can be focused specifically to benefit the 509 people with the greatest need.

510

511

512 Acknowledgments: The authors would like to express their gratitude to the Japan Chronic

513 Disease Self-Management Association, as well as to all of the people who participated in the

514 study. MJ Park is grateful for advice received from Y Yamazaki, for help and collaboration

515 provided by the self-management research team at the University of Tokyo, for administrative

516 assistance and technical support received from N Okamoto, and for advice and academic support 517 received from T Kiuchi and H Ishikawa.

518 


\section{REFERENCES}

520

521 Ahn, S., Basu, R., Smith, M. L., Jiang, L, Lorig, K., Whitelaw, N., Ory, M. G. (2013) The

522

523

524

525

526

527

528

529

530

531

532

533

534

535

536

537

538

539

540 impact of chronic disease self-management programs: healthcare savings through a community-based intervention. BMC Public Health. 13, 1141. doi: 10.1186/1471-245813-1141.

Bandura, A. (2019) Applying Theory for Human Betterment. Perspectives on Psychological Science. 14(1), 12-15. doi: 10.1177/1745691618815165.

Barlow, J., Wright, C., Turner, A., \& Bancroft, G. (2005). A 12-month follow-up study of selfmanagement training for people with chronic disease: Are changes maintained over time? British Journal Of Health Psychology, 10(4), 589-599. doi: 10.1348/135910705x26317

Barlow, J., Turner, A., Swaby, L., Gilchrist, M., Wright, C., \& Doherty, M. (2008). An 8-yr follow-up of Arthritis Self-Management Programme participants. Rheumatology, 48(2), 128-133. doi: 10.1093/rheumatology/ken429

Basu, R., Ory, M. G, Towne, S. D. Jr., Smith, M. L., Hochhalter, A. K., Ahn S. (2015) Costeffectiveness of the chronic disease self-management program: implications for community-based organizations. Frontiers in Public Health. 2015 Apr 27;3:27. doi: 10.3389/fpubh.2015.00027

Brady, T., Murphy, L., O’Colmain, B., Beauchesne, D., Daniels, B., \& Greenberg, M., House, M., \& Chervin, D. (2013). A Meta-Analysis of Health Status, Health Behaviors, and Health Care Utilization Outcomes of the Chronic Disease Self-Management Program. Preventing Chronic Disease, 10. doi: 10.5888/pcd10.120112 
541 Caplin, D., \& Creer, T. (2001). A Self-Management Program for Adult Asthma. III. Maintenance 542 and Relapse of Skills. Journal Of Asthma, 38(4), 343-356. doi: 10.1081/jas-100000263

543 Chronic Disease Self-Management Program (SMRC). (2018). Retrieved from

544 http://patienteducation.stanford.edu/programs/cdsmp.html

545 Clark, N. (2003). Management of Chronic Disease by Patients. Annual Review Of Public Health, 546 24(1), 289-313. doi: 10.1146/annurev.publhealth.24.100901.141021

547 Cook, W., Karriker-Jaffe, K., Bond, J., \& Lui, C. (2015). Asian American Problem Drinking 548 Trajectories During the Transition to Adulthood: Ethnic Drinking Cultures and Neighborhood Contexts. American Journal Of Public Health, 105(5), 1020-1027. doi: 10.2105/ajph.2014.302196

551 Foster, G., Taylor, S. J. C., Eldridge, S., Ramsay, J., Griffiths, C. J. (2007) Self-management 552 education programmes by lay leaders for people with chronic conditions. Cochrane Database of Systematic Reviews 4: Art. No.: CD005108.

554 Franek, J. (2013). Self-management support interventions for persons with chronic disease: an evidence-based analysis. Ontario Health Technology Assessment Series, 13 9, 1-60.

556 Franks, P., Chapman, B., Duberstein, P., \& Jerant, A. (2009). Five factor model personality 557 factors moderated the effects of an intervention to enhance chronic disease management

560 Giacobbi, P., R. (2016) Theoretical, Critical, and Practical Reflections on the Long-Term 561 Maintenance of Health Behavior Change. American Journal of Lifestyle Medicine. 10(6), 377-380. doi: 10.1177/1559827616662435 
563 Glasgow, R., Toobert, D., Hampson, S., \& Strycker, L. (2002). Implementation, generalization

564 and long-term results of the "choosing well" diabetes self-management intervention.

565 Patient Education And Counseling, 48(2), 115-122. doi: 10.1016/s0738-3991(02)00025-3

566 Green, L. (1977). Evaluation and measurement: some dilemmas for health education. American

567 Journal Of Public Health, 67(2), 155-161. doi: 10.2105/ajph.67.2.155

568 Harrell, F. (2019). Dichotomania. http://www.fharrell.com/post/errmed/\#catg Accessed on

$569 \quad$ February 25, 2019

570 Harrison, M., Reeves, D., Harkness, E., Valderas, J., Kennedy, A., Rogers, A., Hann, M., \&

571 Bower, P. (2012). A secondary analysis of the moderating effects of depression and

572 multimorbidity on the effectiveness of a chronic disease self-management programme.

573 Patient Education And Counseling, 87(1), 67-73. doi: 10.1016/j.pec.2011.06.007

574 Haslam, D. (2015). "You're an Expert in Me": The Role of the Generalist Doctor in the

575 Management of Patients with Multimorbidity. Journal Of Comorbidity, 5(1), 132-134.

$576 \quad$ doi: $10.15256 /$ joc. 2015.5 .65

577 Hendershot, C.S., Witkiewitz, K., George, W.H., \& Marlatt, G.A. (2011) Relapse prevention for

578 addictive behaviors. Substance Abuse Treatment, Prevention, and Policy, 6, 17. doi:

$579 \quad 10.1186 / 1747-597 X-6-17$

580 Hennessy, M., Bolan, G., Hoxworth, T., Iatesta, M., Rhodes, F., \& Zenilman, J. (1999). Using

581

582 growth curves to determine the timing of booster sessions. Structural Equation Modeling:

583 Hibbard, J., Mahoney, E., Stock, R., \& Tusler, M. (2007). Do Increases in Patient Activation 584 Result in Improved Self-Management Behaviors? Health Services Research, 42(4), 14431463. doi: 10.1111/j.1475-6773.2006.00669.x 
586 Huang, G. H., \& Bandeen-Roche, K. (2004). Building an identifiable latent class model with 587 covariate effects on underlying and measured variables. Psychometrika, 69, 5-32.

588 Japan Chronic Disease Self-Management Association. (2018). Retrieved from http://www.j589 cdsm.org/

590 Jerant, A., Chapman, B., Duberstein, P., \& Franks, P. (2010). Effects of personality on self-rated 591 health in a 1-year randomized controlled trial of chronic illness self-management. British

592 Journal Of Health Psychology, 15(2), 321-335. doi: 10.1348/135910709x464353

593 Joseph, R. P., Daniel, C. L., Thind, H., Benitez, T. J., \& Pekmezi, D. (2016) Applying

594 Psychological Theories to Promote Long-Term Maintenance of Health Behaviors.

595 American Journal of Lifestyle Medicine. 10(6): 356-368. doi:

596 $10.1177 / 1559827614554594$

597 Karison, E., Lee, I., Cook, N., Manson, J., Buring, J., \& Hennekens, C. (1999). Comparison of 598 Self-Reported Diagnosis of Connective Tissue Disease with Medical Records in Female

599 Health Professionals: The Women's Health Cohort Study. American Journal Of

600 Epidemiology, 150(6), 652-660. doi: 10.1093/oxfordjournals.aje.a010064

601

602

603

604

605

606

607

608

Kozlowski, A.J., Pretz, C.R., Dams-O'Connor, K., Kreider, S., Whiteneck, G. (2013) An introduction to applying individual growth curve models to evaluate change in rehabilitation: a National Institute on Disability and Rehabilitation Research Traumatic Brain Injury Model Systems report. Archives of Physical Medicine and Rehabilitation, 94(3):589-96. doi: 10.1016/j.apmr.2012.08.199.

Krebs, P., Prochaska, J., \& Rossi, J. (2010). A meta-analysis of computer-tailored interventions for health behavior change. Preventive Medicine, 51(3-4), 214-221. doi: 10.1016/j.ypmed.2010.06.004 
609 Lorig, K., \& González, V. (1992). The Integration of Theory with Practice: A 12-Year Case 610 Study. Health Education Quarterly, 19(3), 355-368. doi: 10.1177/109019819201900307

611 Lorig, K., \& Holman, H. (1989). Long-term outcomes of an arthritis self-management study: Effects of reinforcement efforts. Social Science \& Medicine, 29(2), 221-224. doi: $10.1016 / 0277-9536(89) 90170-6$

614 Lorig, K., Holman, H., Sobel, D., Laurent, D., \& González, V. (2001). Living a Healthy Life 615 With Chronic Conditions: Self-management of Heart Disease, Arthritis, Diabetes, Asthma, Bronchitis, Emphysema and Others (Translated by Fusae, K.) (2nd ed.). Tokyo: Nihon Kango Kyokai (in Japanese). Care, 42(4), 346-354. doi: 10.1097/01.mlr.0000118709.74348.65

621 Lorig, K., Ritter, P., Laurent, D., \& Plant, K. (2006). Internet-Based Chronic Disease Self622 Management. Medical Care, 44(11), 964-971. doi: 10.1097/01.mlr.0000233678.80203.c1

623 Lorig, K., Ritter, P., Villa, F., \& Piette, J. (2007). Spanish Diabetes Self-Management With and 624 Without Automated Telephone Reinforcement: Two randomized trials. Diabetes Care, 625 31(3), 408-414. doi: $10.2337 / \mathrm{dc} 07-1313$

Lorig, K., Stewart, A., Ritter, P., González, V., Laurent, D., \& Lynch, J. (1996). Outcome measures for health education and other health care interventions. Thousand Oaks: Sage Publications.

Matsudaira, T., Igarashi, H., Kikuchi, H., Kano, R., Mitoma, H., Ohuchi, K., \& Kitamura, T. 630 (2009). Factor structure of the Hospital Anxiety and Depression Scale in Japanese 
631

632

633 Menon, J., \& Kandasamy, A. (2018) Relapse prevention. Indian Journal of Psychiatry, 60(Suppl

psychiatric outpatient and student populations. Health And Quality Of Life Outcomes, 7(1), 42. doi: 10.1186/1477-7525-7-42

634 4), S473-S478. doi: 10.4103/psychiatry.IndianJPsychiatry_36_18

635

636

637

638

639

640

641

642

643

644

645

646

647

648

649

650

651

652

653 hybrid concept analysis. Nursing Outlook. 63(2), 154-161. doi: 10.1016/j.outlook.2014.07.005

Min, L., Wenger, N., Fung, C., Chang, J., Ganz, D., Higashi, T., Kamberg, C. J., Maclean, C. H., Roth, C. P., Solomon, D. H., Young, R. T., \& Reuben, D. B. (2007). Multimorbidity is Associated With Better Quality of Care Among Vulnerable Elders. Medical Care, 45(6), 480-488. doi: 10.1097/mlr.0b013e318030fff9

Moynihan, R., Henry, D., \& Moons, K. (2014). Using Evidence to Combat Overdiagnosis and Overtreatment: Evaluating Treatments, Tests, and Disease Definitions in the Time of Too Much. Plos Medicine, 11(7), e1001655. doi: 10.1371/journal.pmed.1001655

Muthén, B. (2004). Latent variable analysis: Growth mixture modeling and related techniques for longitudinal data. In D. Kaplan (Ed.), The Sage handbook of quantitative methodology for the social sciences (pp. 345-368). London: SAGE.

Newman, S., Steed, L., \& Mulligan, K. (2009). Chronic physical illness (p. 72). Maidenhead: Open University Press.

Nguyen, H., Carrieri-Kohlman, V., Rankin, S., Slaughter, R., \& Stulbarg, M. (2005). Is Internetbased support for dyspnea self-management in patients with chronic obstructive pulmonary disease possible? Results of a pilot study. Heart \& Lung: The Journal Of Acute And Critical Care, 34(1), 51-62. doi: 10.1016/j.hrtlng.2004.06.005 
654 Nolte, S., Elsworth, G., Sinclair, A., \& Osborne, R. (2007). The extent and breadth of benefits 655 from participating in chronic disease self-management courses: A national patient656

657 reported outcomes survey. Patient Education And Counseling, 65(3), 351-360. doi:

658 Norris, S., Lau, J., Smith, S., Schmid, C., \& Engelgau, M. (2002). Self-Management Education 659 for Adults With Type 2 Diabetes: A meta-analysis of the effect on glycemic control.

O'Connell, S., McCarthy, V. J. C., Savage, E. (2018) Frameworks for self-management support for chronic disease: a cross-country comparative document analysis. BMC Health Services Research, 25;18(1):583. doi: 10.1186/s12913-018-3387-0.

665

Park, M., Green, J., Ishikawa, H., Yamazaki, Y., Kitagawa, A., \& Ono, M., Yasukata, F., \& 666 Kiuchi, T. (2013). Decay of Impact after Self-Management Education for People with Chronic Illnesses: Changes in Anxiety and Depression over One Year. Plos ONE, 8(6), e65316. doi: 10.1371/journal.pone.0065316

Park, M., Green, J., Ishikawa, H., \& Kiuchi, T. (2012). Hidden decay of impact after education for self-management of chronic illnesses: hypotheses. Chronic Illness, 9(1), 73-80. doi: $10.1177 / 1742395312453351$

Park, Y. S., Xing, K., \& Lee, Y. S. (2018). Explanatory cognitive diagnostic models: Incorporating latent and observed predictors. Applied Psychological Measurement, 42(5), 376-392.

675

Rabe-Hesketh, S., Skrondal, A., \& Pickles, A. (2004). Generalized multilevel structural equation modeling. Psychometrika, 69(2), 167-190. doi: 10.1007/bf02295939 
676 Ram, N., \& Grimm, K. (2009). Methods and Measures: Growth mixture modeling: A method for 677 identifying differences in longitudinal change among unobserved groups. International 678 Journal Of Behavioral Development, 33(6), 565-576. doi: 10.1177/0165025409343765

679

680

681

682

683

684

685

686

687

688

689

690

691

692

693

694

695

696

697

Richardson, G., \& Rogers, A. (2008). Predicting who will benefit from an Expert Patients Programme self-management course. British Journal Of General Practice, 58(548), 198203. doi: $10.3399 /$ bjgp08x277320

Riemsma, R., Taal, E., \& Rasker, J. (2003). Group education for patients with rheumatoid arthritis and their partners. Arthritis \& Rheumatism, 49(4), 556-566. doi: 10.1002/art. 11207

Rothman AJ. (2000) Toward a theory-based analysis of behavioral maintenance. Health Psychology. 19(1 Suppl): 64-69.

Sanchez-Villegas, A., Schlatter, J., Ortuno, F., Lahortiga, F., Pla, J., Benito, S., \& MartinezGonzalez, M. A. (2008). Validity of a self-reported diagnosis of depression among participants in a cohort study using the Structured Clinical Interview for DSM-IV (SCIDI). BMC Psychiatry, 8(1), 43.

Schulman-Green, D., Jaser, S. S., Park, C., \& Whittemore, R. (2016). A metasynthesis of factors affecting self-management of chronic illness. Journal of Advanced Nursing, 72(7), 14691489.

Sciamanna, C. N., Kiernan, M., Rolls, B. J., Boan, J., Stuckey, H., Kephart, D., Miller, C. K., Jensen, G., Hartmann, T. J., Loken, E., Hwang, K. O., Williams, R. J., Clark, M. A., Schubart, J. R., Nezu, A. M., Lehman, E., \& Dellasega, C. (2011) Practices associated 
698

699

700

701

702

703

704

705

706

707

708

709

710

711

712

713

714

715

716

717

718

with weight loss versus weight-loss maintenance. American Journal of Preventive Medicine. 41(2), 159-66. doi: 10.1016/j.amepre.2011.04.009.

Smeulders, E., van Haastregt, J., Ambergen, T., Stoffers, H., Janssen-Boyne, J., Uszko-Lencer, N., Gorgels, A., Lodewijks-van der Bolt, C., van Eijk, J., \& Kempen, G. (2010). Heart failure patients with a lower educational level and better cognitive status benefit most from a self-management group programme. Patient Education And Counseling, 81(2), 214-221. doi: 10.1016/j.pec.2010.01.003

Swerissen, H., Belfrage, J., Weeks, A., Jordan, L., Walker, C., Furler, J., McAvoy, B., Carter, M., \& Peterson, C. (2006). A randomised control trial of a self-management program for people with a chronic illness from Vietnamese, Chinese, Italian and Greek backgrounds. Patient Education And Counseling, 64(1-3), 360-368. doi: 10.1016/j.pec.2006.04.003

Wada, K., Yatsuya, H., Ouyang, P., Otsuka, R., Mitsuhashi, H., Takefuji, S., Matsushita, K., Sugiura, K., Hotta, Y., Toyoshima, H., \& Tamakoshi, K. (2009). Self-reported medical history was generally accurate among Japanese workplace population. Journal Of Clinical Epidemiology, 62(3), 306-313. doi: 10.1016/j.jclinepi.2008.04.006

Whitelaw, N., Lorig, K., Smith, M., \& Ory, M. (2013). Webinar: Findings from the CDSMP National Study. Retrieved from https:/www.ncoa.org/resources/webinar-findings-fromthe-cdsmp-national-study/

Witkiewitz K and Marlatt GA. Relapse prevention for alcohol and drug problems: that was Zen, this is Tao. American Psychologist 2004; 59: 224-235. 


\section{Figure 1}

Trajectories of change after health education, showing two trajectory-defined groups for each of the three outcomes

Growth-Mixture Modeling revealed two trajectory-defined groups for each outcome. On anxiety and depression higher scores are worse. On communication with physicians higher scores are better. For each outcome, one of those two groups had improvement followed by deterioration: decay of impact. For Anxiety and Depression, a score of 9 is the cutoff used in Japan to separate non-cases from possible and probable cases.
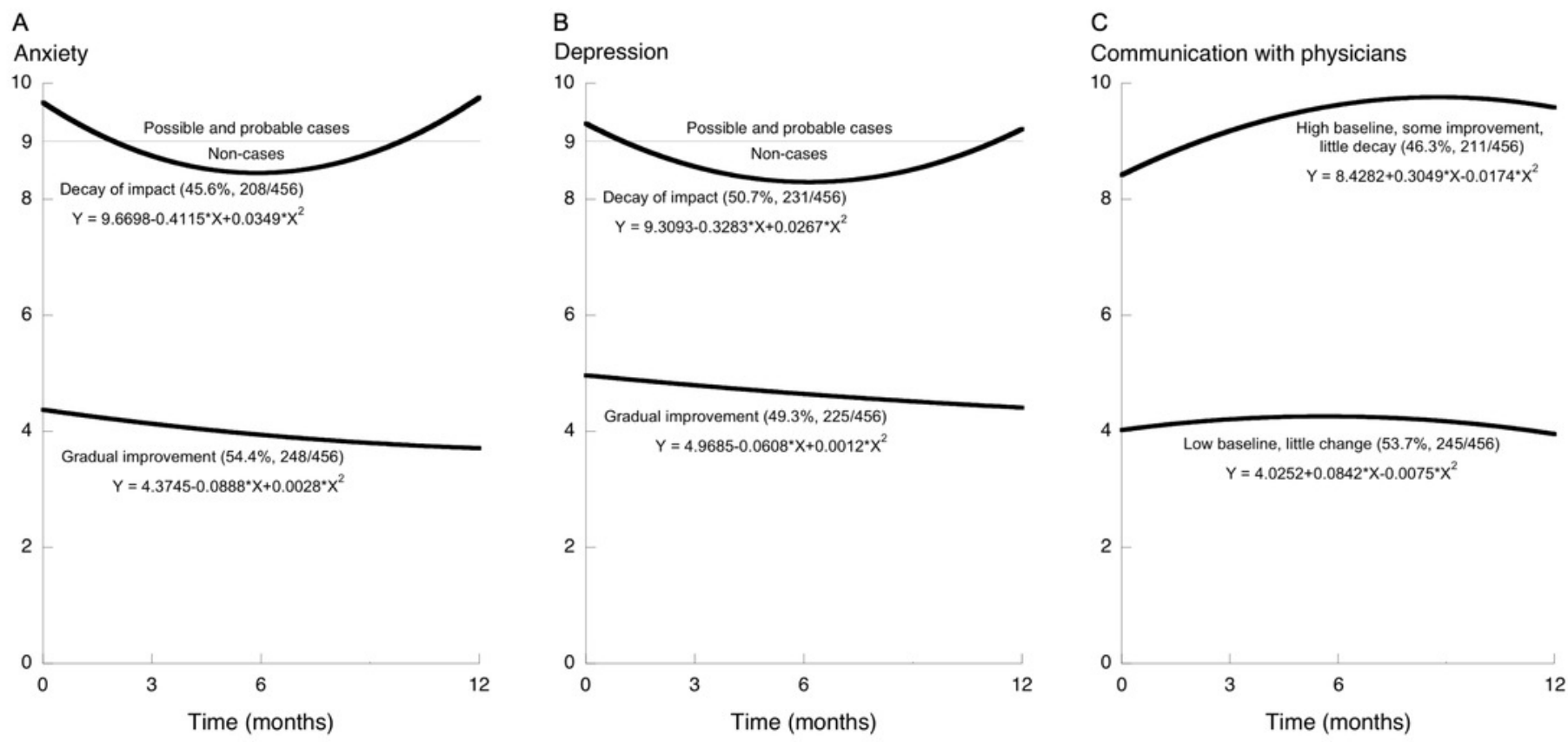


\section{Table $\mathbf{1}$ (on next page)}

Descriptive statistics for all CDSMP participants considered together, baseline and follow up over one year

The outcomes discussed here are anxiety, depression, and communication with physicians. Self-efficacy was used as a mediator in subsequent analyses because of its importance in the theoretical basis of the CDSMP. 
1

\begin{tabular}{lcccccccccccc}
\hline \multirow{2}{*}{ Factor } & \multicolumn{3}{c}{ Baseline } & \multicolumn{2}{c}{ 3 Months } & \multicolumn{3}{c}{ 6 Months } \\
\cline { 2 - 10 } & $n$ & Mean & SD & $n$ & Mean & SD & $n$ & Mean & SD & $n$ & Mean & SD \\
\hline $\begin{array}{l}\text { Self-efficacy: 0-60, } \\
\text { higher scores are better }\end{array}$ & 456 & 32.33 & 12.54 & 415 & 34.48 & 12.02 & 425 & 35.16 & 12.10 & 404 & 35.14 & 12.99 \\
\hline $\begin{array}{l}\text { Anxiety: 0-21, } \\
\text { lower scores are better }\end{array}$ & 456 & 6.89 & 4.25 & 423 & 6.27 & 4.00 & 428 & 6.03 & 4.22 & 406 & 6.43 & 4.62 \\
$\begin{array}{l}\text { Depression: 0-21, } \\
\text { lower scores are better }\end{array}$ & 456 & 7.21 & 3.82 & 423 & 6.69 & 3.64 & 428 & 6.53 & 3.93 & 406 & 6.77 & 4.20 \\
$\begin{array}{l}\text { Communication: 0-15, } \\
\text { higher scores are better }\end{array}$ & 456 & 6.22 & 3.77 & 422 & 6.59 & 3.99 & 426 & 6.91 & 4.11 & 401 & 6.77 & 4.18 \\
\hline
\end{tabular}

2 


\section{Table 2 (on next page)}

Results of growth-curve analysis, all CDSMP participants considered together $(n=456)$

\section{Notes:}

1. Quadratic growth curve fit to data using full-information maximum likelihood (FIML) estimation.

2. Random effects indicate the variability in the fixed effect. For example, the $95 \%$ confidence interval for the slope (Time) of Anxiety is (from -.53 to .05$)=($ Fixed-effect estimate for Time) \pm (Random-effect SD for Time $\times 1.96)=-.24 \pm(.15 \times 1.96)$.

3. Interaction terms with time indicate change in outcome for the time-invariant factor (i.e., male, college, partnered, number of diagnoses, and disease history) over time. 


\begin{tabular}{|c|c|c|c|c|c|c|c|c|c|}
\hline \multirow{2}{*}{ Factor } & \multicolumn{3}{|c|}{ Anxiety } & \multicolumn{3}{|c|}{ Depression } & \multicolumn{3}{|c|}{ Communication } \\
\hline & Coefficient & Std Err & $p$-value & Coefficient & Std Err & $p$-value & Coefficient & Std Err & $p$-value \\
\hline \multicolumn{10}{|l|}{ Fixed effect } \\
\hline Time & -.24 & $(.07)$ & .001 & -.14 & $(.07)$ & .034 & .20 & $(.06)$ & .001 \\
\hline Time $x$ Time & .01 & $(.00)$ & .001 & .01 & $(.00)$ & .038 & -.01 & $(.00)$ & .002 \\
\hline Self-Efficacy & -.10 & $(.01)$ & $<.001$ & -.11 & $(.01)$ & $<.001$ & .03 & $(.01)$ & $<.001$ \\
\hline Male & -.06 & (.41) & .887 & .84 & $(.36)$ & .018 & -.33 & (.44) & .450 \\
\hline College Educated & -.97 & $(.34)$ & .004 & -.04 & $(.30)$ & .882 & .41 & $(.34)$ & .222 \\
\hline Partnered & -.02 & $(.34)$ & .947 & -.23 & $(.30)$ & .443 & .02 & $(.34)$ & .956 \\
\hline Number of Diagnoses & .37 & (.16) & .019 & .18 & (.13) & .190 & .61 & (.15) & $<.001$ \\
\hline History (years) & -.02 & $(.01)$ & .164 & -.04 & $(.01)$ & .004 & -.01 & (.01) & .643 \\
\hline Male x Time & .01 & $(.04)$ & .852 & -.05 & $(.04)$ & .163 & -.03 & $(.04)$ & .366 \\
\hline College $\mathrm{x}$ Time & .03 & $(.03)$ & .328 & -.01 & $(.03)$ & .655 & -.02 & (.03) & .424 \\
\hline Partnered x Time & -.04 & $(.03)$ & .240 & .01 & $(.03)$ & 648 & .01 & $(.03)$ & 688 \\
\hline Number of Diagnoses x Time & .03 & $(.01)$ & .062 & .02 & $(.01)$ & .174 & -.01 & $(.01)$ & .300 \\
\hline History (years) x Time & .00 & $(.00)$ & .267 & .00 & $(.00)$ & .397 & .00 & $(.00)$ & .776 \\
\hline Intercept & 1.19 & $(.57)$ & $<.001$ & 1.93 & $(.48)$ & $<.001$ & 3.98 & (.48) & $<.001$ \\
\hline \multicolumn{10}{|l|}{ Random effect } \\
\hline SD(Time) & .15 & $(.03)$ & & .14 & $(.02)$ & & .14 & $(.02)$ & \\
\hline SD(Intercept) & 2.83 & (.12) & & 2.26 & (.11) & & 3.14 & (.11) & \\
\hline SD(Residual) & 2.64 & $(.08)$ & & 2.45 & $(.08)$ & & 2.17 & $(.08)$ & \\
\hline
\end{tabular}




\section{Table 3(on next page)}

Factors predicting membership in groups defined by their trajectory after the CDSMP

\section{Notes:}

1. Values in parenthesis are standard errors.

2. The results shown are from logistic regression. The 0 -1 coding of group membership (which is the dependent variable) reflects the relative magnitudes of the baseline scores. For all outcomes, the group with the lower baseline score is coded " 0 " and the group with the higher baseline score is coded " 1 ." Thus, the group with less anxiety at baseline is coded " 0 " while the group with more anxiety at baseline is coded "1." The same is true for depression. In contrast, the group with better communication (higher scores) at baseline is coded " 1 " and the group with worse communication (lower scores) at baseline is coded "0." 


\begin{tabular}{|c|c|c|c|c|c|c|}
\hline \multirow{2}{*}{ Factor } & \multicolumn{2}{|l|}{ Anxiety } & \multicolumn{2}{|l|}{ Depression } & \multicolumn{2}{|c|}{ Communication } \\
\hline & Adjusted Odds Ratio & $p$-value & Adjusted Odds Ratio & $p$-value & Adjusted Odds Ratio & $p$-value \\
\hline Male & $.92 \quad(.23)$ & .730 & 1.50 & .112 & $.62 \quad(.15)$ & .050 \\
\hline College & $.59 \quad(.12)$ & .009 & $(.17)$ & .376 & $(.23)$ & .432 \\
\hline Partnered & $(.20)$ & .947 & $(.16)$ & .227 & $(.23)$ & .418 \\
\hline Self-Efficacy & $.97 \quad(.01)$ & $<.001$ & $(.01)$ & $<.001$ & $(.01)$ & .013 \\
\hline Number of Diagnoses & $1.23 \quad(.12)$ & .032 & $(.11)$ & .257 & $(.13)$ & .006 \\
\hline History (years) & $(.01)$ & .266 & $(.01)$ & .244 & $(.01)$ & .683 \\
\hline Intercept & $2.66 \quad(.99)$ & .009 & $(2.49)$ & $<.001$ & $(.11)$ & .001 \\
\hline
\end{tabular}

